\title{
Neurodevelopmental epigenetic etiologies: insights from studies on mouse models of fetal alcohol
} spectrum disorders

\section{"A small but growing number of fetal alcohol spectrum disorder researchers have similarly hypothesized that alcohol may exert its effects on fetal development through epigenetic mechanisms."}

\section{KEYWORDS: alcohol " disease of developmental origin $\approx$ fetal alcohol syndrome n neurodevelopment = systems biology}

The development of an organism from a single cell (ontogeny) is an intricate and enigmatic process. It is initiated by maternal and paternal nuclear contributions that are complementary but not identical. The phenotypic outcome of the child is not only dependent on traditional genetic inheritance, but also on the prenatal environment, including the various exogenous and endogenous signals. Environmental factors affect various pre- and post-natal developmental processes via epigenetic regulation of gene expression. As such, many genetically complex disorders are studied at the epigenetic level. This approach has led to a much deeper understanding of a host of diseases and disorders from cancer to schizophrenia. A small but growing number of fetal alcohol spectrum disorder (FASD) researchers have similarly hypothesized that alcohol may exert its effects on fetal development through epigenetic mechanisms.

Individuals afflicted with FASD exhibit impairments in cognition, learning, executive function, judgment, attention and social adaptation [1,2]. The extent to which an individual exhibits such impairments depends on their genetic background, as well as the timing and dose of alcohol exposure [3,4]. FASD affects 2-5\% of pregnancies in North America, and has an annual cost in the tens of billions of dollars, making it a key public health concern $[5,6]$.

Using various animal models and treatment regimes, a variety of the effects of alcohol on the brain epigenome have been studied, including genome-wide hypomethylation [7], and genespecific hyper- and hypo-methylation [8-10], some of which are coincident with changes in gene expression and persist into adulthood. Furthermore, epigenetic and gene expression changes are associated with FASD-related endophenotypes at multiple developmental time points [4,11-13]. These changes result in a long-lasting epigenetic footprint that can lead to changes in gene expression and behaviors characteristic of FASD. The results of our research and that of other groups suggest that prenatal alcohol exposure during any stage of pregnancy can result in the development of FASD [14].

Our group has been interested in the molecular basis of FASD for some time. We began by creating a mouse model of the behavioral abnormalities associated with FASD [4,11]. We then began to explore the molecular mechanisms underlying associated endophenotypes $[3,12]$. We have used four treatment protocols to examine the effects of binge drinking (intraperitoneal injection) during the first, second or third trimester of pregnancy, as well as moderate voluntary alcohol consumption throughout pregnancy. We found that all four exposure paradigms produced long-lasting (up to 70 days of age) changes in miRNA and gene expression [3]. Interestingly, changes in some miRNAs matched specific dysregulated target genes that are involved in a number of neurodevelopmental processes and adult brain functions. Interestingly, none of the identified transcripts were seen in all four paradigms that represented alcohol exposure at different stages of neurodevelopment. The exception was H/MBII-52, a snoRNA with a role in the etiology of numerous neurological disorders. We also examined DNA methylation in the voluntary alcohol consumption paradigm and found long-lasting changes in $>6500$ gene promoters. The affected genes are involved in neurodevelopment and adult brain processes. The methylation changes also included a number of CTCF binding sites that are known to bind to differentially methylated regions of imprinted genes, among others [15]. These changes are relatively stable during

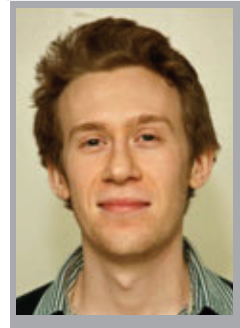

Benjamin I Laufer Molecular Genetics Unit, Department of Biology, Western University, London, ON, N6A 5B7, Canada

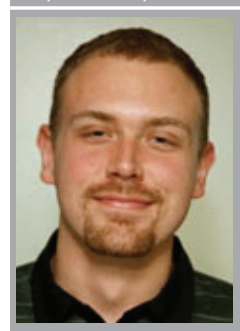

Eric J Diehl

Molecular Genetics Unit, Department of Biology, Western University, London, ON, N6A 5B7, Canada

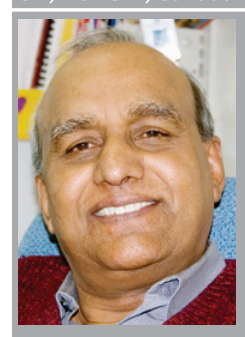

Shiva M Singh

Author for correspondence: Molecular Genetics Unit, Department of Biology, Western University, London, ON, N6A 5B7, Canada Tel.: +15196613135 Fax. +15196613935 ssingh@uwo.ca 
development and must undergo reprogramming in germ cells. The results offer a number of novel insights.

FASD may develop as a long-lasting and novel gene expression footprint with implications in alcohol-related cellular functions. Our results argue for a continuum of changes from gene expression to cellular physiology, neurodevelopment and FASD-related behaviors. Although this genotype-phenotype correlation may partially explain the effect of alcohol, it does not address the mechanism associated with altered, stable and long-lasting expression of selected genes following alcohol exposure during neurodevelopment. We argue that these changes are accommodated by epigenetic mechanisms that include DNA methylation, miRNA and histone modifications.

\section{"...neurodevelopmental epigenetic alterations following prenatal alcohol exposure are an integral part of the etiology of fetal alcohol spectrum and related disorders."}

Much of our understanding of FASD has come from experiments that take a reductionist approach to disease etiology. Reductionism is a philosophy that the understanding of a complex system can be achieved in full by understanding its simpler component parts. Systems biology, on the other hand, relies on examining the entirety of cellular processes and interactions in concert [16]. While a reductionist approach can quite successfully identify genetic components and interactions, it offers no method of understanding large-scale emergent system properties; "the pluralism of causes and effects in biological networks is better addressed by observing, through quantitative measures, multiple components simultaneously and by rigorous data integration with mathematical models" [17]. A systems biology approach has been applied to many complex diseases [18]; however, to date, no study has yet taken a largescale systems approach to examining fetal alcohol exposure. This is chiefly due to a lack of the necessary comprehensive data. To this end, we have begun to take steps toward a systems biology approach to FASD in our most recent experiments [13].

Most epigenetic studies of FASD have focused on one or several genetic loci [9,19-22]. We have extended such studies to assess genome-wide DNA methylation and miRNA in a mouse model [13]. We believe that the integration of multiple data sets at multiple levels will be a key step in moving FASD research forward. By examining data from multiple gene expression microarrays from multiple alcohol exposure models, we have gained a higher level understanding of alcohol's effect on the brain transcriptome. Our results have highlighted the importance of studying genes as inter- and intra-related modular components responding to the environment [23]. While single-gene analysis has provided a solid foundation for preliminary research into complex traits, it can exclude important, but subtle, effects on cellular pathways. Indeed, many cellular processes affect sets of genes acting in concert. A 1.2-fold increase in many genes of a pathway can have a potentially greater physiological impact than a 20 -fold increase in a single gene. Using a pathway analysis based on the principles of independent component analysis [24] our observations of single genes showing low-fold changes and borderline statistical significance have much greater statistical significance and biological impact when grouped together according to modular functions that show a significant enrichment.

\section{"...epigenetic and gene expression changes are associated with fetal alcohol spectrum disorder-related endophenotypes at multiple developmental time points."}

Nevertheless, the scale-free topology of cellular networks allows for some key individual genes to have crucial properties [25]. The distribution of nodes (i.e., genes) in cellular networks is highly nonuniform with most of the nodes having only a few links to other nodes. However, there are a few nodes with a very large number of links called hubs. The importance of the relationship between a system and single gene is highlighted in these cases. While a network can tolerate many disruptions to its lesser-connected nodes, a similar disruption to a single node can be catastrophic to the networks it connects. This principle is highlighted in our results by the disruption in PTEN signaling. We observed changes in key hubs of this pathway, such as the increased methylation in $A K T$ and increased expression of PTEN. Interestingly, these two genes act in opposing fashions and alterations can have profound effects on both neurodevelopment and neuroplasticity [26]. Given these facts we hypothesize that the changes observed in these hubs play a critical role in the observed molecular footprint of 
altered methylation across half of the members of this signaling pathway.

These findings also unite two mechanisms behind fetal alcohol exposure, epigenetics and reactive oxygen species. Reactive oxygen species signaling has a significant role in the functions of neurodevelopment and plasticity; it interacts with the hubs of PTEN signaling by using reversible oxidation to alter protein function [27]. These findings reveal an intimate relationship between two molecular mechanisms of FASD. In our opinion, this approach is a significant contribution to our understanding of FASD. It allows for a more complete annotation of the pool of endophenotypes associated with FASD, provides valuable biomarkers given the dynamic and theoretically reversible nature of the epigenome, and finally it begins to put together the puzzle pieces of how exactly the epigenome serves as the bridge between nature and nurture for shaping complex traits, such as brain function [28].

Moving forward, several conclusions and principles can be drawn from our research to date. First, our data argue that neurodevelopmental epigenetic alterations following prenatal alcohol exposure are an integral part of the etiology of fetal alcohol spectrum and related disorders. Our results offer a novel paradigm in appraising the role of environmental factors in complex diseases in general and neurodevelopmental diseases in particular. Furthermore, they support an approach that incorporates interactions at every level, particularly the epigenome. The true significance of epigenetics comes from its expansion of the genetic code from a combination of just four base pairs to a much more complex code that can consist of combinations of hundreds of molecular marks that are in unique combinations both spatially and temporally. Similarly, the resulting observations on the extent of genetic and epigenetic variations affecting a relatively large number of genes argue for a genome-wide approach that relies on systems biology. It takes into account pathways that are ultimately affected both spatially and temporally. We feel that it better reflects the complexity of most biological systems. Finally, given the dynamic nature of epigenetic marks, there remains a great potential for therapeutic reversals of the long-lasting changes that contribute to diseases of epigenetic etiology, including FASD.

\section{Financial \& competing interests disclosure \\ The authors have no relevant affiliations or financial involvement with any organization or entity with a finan- cial interest in or financial conflict with the subject matter or materials discussed in the manuscript. This includes employment, consultancies, honoraria, stock ownership or options, expert testimony, grants or patents received or pending, or royalties. \\ No writing assistance was utilized in the production of this manuscript.}

\section{References}

1 Jirikowic T, Olson HC, Kartin D. Sensory processing, school performance, and adaptive behavior of young school-age children with fetal alcohol spectrum disorders. Phys. Occup. Ther. Pediatr. 28(2), 117-136 (2008).

2 Herman LE, Acosta MC, Chang PN. Gender and attention deficits in children diagnosed with a fetal alcohol spectrum disorder. Can. J. Clin. Pharmacol. 15(3), e411-e419 (2008).

3 Kleiber ML, Mantha K, Stringer RL, Singh SM. Neurodevelopmental alcohol exposure elicits long-term changes to gene expression that alter distinct molecular pathways dependent on timing of exposure. J. Neurodev. Disord. 5(1), 6 (2013).

4 Mantha K, Kleiber M, Singh S. Neurodevelopmental timing of ethanol exposure may contribute to observed heterogeneity of behavioral deficits in a mouse model of fetal alcohol spectrum disorder (FASD). Behav. Brain Sci. 3, 85-99 (2013).
5 May PA, Gossage JP, Kalberg WO et al. Prevalence and epidemiologic characteristics of FASD from various research methods with an emphasis on recent in-school studies. Dev. Disabil. Res. Rev. 15(3), 176-192 (2009).

6 Popova S, Stade B, Bekmuradov D, Lange S, Rehm J. What do we know about the economic impact of fetal alcohol spectrum disorder? A systematic literature review. Alcohol Alcohol. 46(4), 490-497 (2011).

7 Garro AJ, McBeth DL, Lima V, Lieber CS. Ethanol-consumption inhibits fetal DNA methylation in mice: implications for the fetal alcohol syndrome. Alcohol Clin. Exp. Res. 15(3), 395-398 (1991).

8 Liu Y, Balaraman Y, Wang G, Nephew KP, Zhou FC. Alcohol exposure alters DNA methylation profiles in mouse embryos at early neurulation. Epigenetics 4(7), 500-511 (2009).

9 Kaminen-Ahola N, Ahola A, Maga M et al. Maternal ethanol consumption alters the epigenotype and the phenotype of offspring in a mouse model. PLoS Genet. 6(1), e1000811 (2010).
10 Bekdash RA, Zhang C, Sarkar DK. Gestational choline supplementation normalized fetal alcohol-induced alterations in histone modifications, DNA methylation, and proopiomelanocortin (POMC) gene expression in $\beta$-endorphin-producing pomc neurons of the hypothalamus. Alcohol. Clin. Exp. Res. 37(7), 1132-1142 (2013).

11 Kleiber ML, Wright E, Singh SM. Maternal voluntary drinking in C57BL/6J mice: advancing a model for fetal alcohol spectrum disorders. Behav. Brain Res. 223(2), 376-387 (2011).

12 Kleiber ML, Laufer BI, Wright E, Diehl EJ, Singh SM. Long-term alterations to the brain transcriptome in a maternal voluntary consumption model of fetal alcohol spectrum disorders. Brain Res. 1458, 18-33 (2012).

13 Laufer BI, Mantha K, Kleiber ML, Diehl EJ, Addison SM, Singh SM. Long lasting alterations to DNA methylation and ncRNAs may underlie the effects of fetal alcohol exposure. Dis. Model. Mech. 6(4), 977-992 (2013). 
14 May PA, Gossage JP. Estimating the prevalence of fetal alcohol syndrome - a summary. Alcohol Res. Health 25(3), 159-167 (2001).

15 Lin S, Ferguson-Smith AC, Schultz RM, Bartolomei MS. Nonallelic transcriptional roles of ctcf and cohesins at imprinted loci. Mol. Cell. Biol.31(15), 3094-3104 (2011).

16 Lim WA, Lee CM, Tang C. Design principles of regulatory networks: searching for the molecular algorithms of the cell. Mol. Cell 49(2), 202-212 (2013).

17 Sauer U, Heinemann M, Zamboni N. Getting closer to the whole picture. Science 316(5824), 550-551 (2007).

18 Silverman EK, Loscalzo J. Network medicine approaches to the genetics of complex diseases. Discov. Med. 14(75), 143-152 (2012).

19 Sathyan P, Golden HB, Miranda RC. Competing interactions between microRNAs determine neural progenitor survival and proliferation after ethanol exposure: evidence from an ex vivo model of the fetal cerebral cortical neuroepithelium. J. Neurosci. 27(32), 8546-8557 (2007).

20 Haycock PC, Ramsay M. Exposure of mouse embryos to ethanol during preimplantation development: effect on DNA methylation in the H19 imprinting control region. Biol. Reprod. 81(4), 618-627 (2009).

21 Wang LL, Zhang Z, Li Q et al. Ethanol exposure induces differential microRNA and target gene expression and teratogenic effects which can be suppressed by folic acid supplementation. Hum. Reprod. 24(3), 562-579 (2009).

22 Kaminen-Ahola N, Ahola A, Flatscher-Bader $\mathrm{T}$ et al. Postnatal growth restriction and gene expression changes in a mouse model of fetal alcohol syndrome. Birth Defects Res. Part A Clin. Mol. Teratol. 88(10), 818-826 (2010).

23 Subramanian A, Tamayo P, Mootha VK et al. Gene set enrichment analysis: a knowledge-based approach for interpreting genome-wide expression profiles. Proc. Natl Acad. Sci. USA 102(43), 15545-15550 (2005).

24 Lee SI, Batzoglou S. Application of independent component analysis to microarrays. Genome Biol. 4(11), R76 (2003).

25 Barabasi AL, Oltvai ZN. Network biology: understanding the cell's functional organization. Nat. Rev. Genet. 5(2), 101-113 (2004).

26 Amiri A, Cho W, Zhou J et al. PTEN deletion in adult hippocampal neural stem/progenitor cells causes cellular abnormalities and alters neurogenesis. J. Neurosci. 32(17), 5880-5890 (2012).

27 Ostrakhovitch EA, Semenikhin OA. The role of redox environment in neurogenic development. Arch. Biochem. Biophys. 534(1-2), 44-54 (2013).

28 Laufer B, Singh S. A macro role for imprinted clusters of microRNAs in the brain. MicroRNA 1, 59-64 (2012). 